\title{
Development of a versatile in vitro method for understanding the migration of Fasciola hepatica newly excysted juveniles
}

\author{
ANDRES GARCIA-CAMPOS ${ }^{1,2}$, ALAN W. BAIRD ${ }^{1,2}$ and GRACE MULCAHY ${ }^{1,2}$ \\ ${ }^{1}$ School of Veterinary Medicine, University College Dublin, Veterinary Sciences Centre, Belfield, Dublin 4, Ireland \\ ${ }^{2}$ Conway Institute of Biomedical and Biomolecular Research, University College Dublin, Belfield, Dublin 4, Ireland
}

(Received 24 Fuly 2015; revised 6 October 2015; accepted 8 October 2015; first published online 2 November 2015)

SUMMARY

Fasciola hepatica is a parasitic trematode that causes serious losses to livestock producers, and also zoonotic disease. The limitations of chemotherapy for the control of fasciolosis have led to significant interest in the development of vaccines to protect cattle and sheep from infection. However, relatively few studies have concentrated on the mechanisms of invasion of the gut by newly excysted juvenile liver flukes (NEJ) and the host response triggered by this event. The aim of this work was to develop an in vitro model to study invasion by NEJ, while also reducing the requirement for challenge infections of experimental animals. Fasciola hepatica metacercariae were excysted in vitro and placed into compartments containing rat distal jejunal sheets. Variations in incubation medium, chamber size and incubation temperature were used to identify optimal conditions for NEJ migration across the gut. Histological examination showed increased migration until 120 min post-incubation. The use of RPMI, without gassing at $39^{\circ} \mathrm{C}$, as the incubation medium was found to be optimal, with $40.5 \%$ of NEJ migrating after $150 \mathrm{~min}$. This study describes a readily-reproducible method for studying the migration of $F$. hepatica NEJ within the definitive host. It will be useful for identifying potential drug and vaccine targets.

Key words: Fasciola hepatica, NEJ, gut, jejunum, method optimization, horizontal diffusion chamber.

\section{INTRODUCTION}

Fasciola hepatica is a trematode, distributed worldwide, with a high economic impact in the livestock industry. Cattle and sheep become infected by ingestion of metacercariae that excyst in the digestive tract, releasing the newly excysted juveniles (NEJ), which are capable of burrowing through the intestine and migrating to the liver through the peritoneal cavity. Relatively little work has been done to understand the mechanisms through which NEJ orchestrate invasion and the host response that they trigger at gut level. Most of the existing literature dates from 1970s to the 1990s (Hanna and Ballawy, 1975; Van Milligen et al. 1999) with only one publication in the last decade (McGonigle et al. 2008). Just after NEJ excystation, the NEJ are able to recognize the intestinal environment, changing their behaviour in the presence of duodenal extracts (Sukhdeo and Mettrick, 1986). They also have the ability to detect the presence of bile salts, triggering migration through the bowel (Tielens et al. 1981). The NEJ excretory/secretory (ES) products comprise a cocktail of important molecules for tissue degradation, feeding and immunoregulation including those belonging to the cathepsin clade. The upregulation and secretion of cathepsin Bs and L3, which have high collagenase activity, is particularly

* Corresponding author: School of Veterinary Medicine, University College Dublin, Veterinary Sciences Centre, Belfield, Dublin 4, Ireland. E-mail: andres.garcia-campos@ ucdconnect.ie important, in view of the necessity for the NEJ to migrate across the intestine (Robinson et al. 2009).

The earliest study reported on NEJ migration is that of Schumacher (1938) who observed the $24 \mathrm{~h}$ period following infection of guinea pigs. Since then, it has been shown in vitro that NEJ have the ability to penetrate rat mid-small intestine (Doy and Hughes, 1981), rat small and large intestine (Hanna and Ballawy, 1975; Kawano et al. 1991; McGonigle et al. 2008) and small and large intestine of other species like mice and rabbits (Kawano et al. 1991). The percentages of successful NEJ migration found in the different models vary from $37 \%$ (Kawano et al. 1991) to 77\% (Van Milligen et al. $1998 a, b)$. A similar NEJ migration rate was reported by Hanna and Ballawy (1975), with Fasciola gigantica $\mathrm{NEJ}$ for $5 \mathrm{~h}$ in vitro. However, when the same experiment was performed in chickens, minimum NEJ invasion was reported (Kawano et al. 1991). Most of the experiments employed for NEJ invasion assessment required the use of various in vitro methods based on large portions of intestine or intestinal loops where screening of the whole sample was difficult because of the large volume to examine. Therefore, the localization of the NEJ within the different mucosal compartments or gut layers remained unknown. An ex vivo model was developed by Van Milligen et al. (1998b), which was useful to clarify both the dynamics of $F$. hepatica migration and the mucosal immunity that naïve and primed infected rats developed after infection (Van Milligen et al. 1998a, b). This method had the

Parasitology (2016), 143, 24-33. C Cambridge University Press 2015. This is an Open Access article, distributed under the terms of the Creative Commons Attribution licence (http://creativecommons.org/licenses/by/4.0/), which permits unrestricted re-use, distribution, and reproduction in any medium, provided the original work is properly cited. 
advantage that the blood supply in the gut was guaranteed, providing conditions more akin to those in vivo. However, the use of ex vivo models is ethically more difficult to justify than in vitro alternatives.

The mechanisms that NEJ employ for interacting with the gut epithelium prior to penetration and the involvement of glycans and $C$-type lectin receptors are still unclear. The aim of this work was to develop a more ethical, robust and efficient in vitro model for studies on the early stages of $F$. hepatica invasion that minimize the requirement for live animals and that will be valuable in understanding these mechanisms.

\section{MATERIAL AND METHODS}

\section{Reagents}

All chemical and reagents were purchased from Sigma-Aldrich Company Ltd. (Ireland) unless otherwise stated.

\section{Excystation of metacercariae in vitro}

Fasciola hepatica metacercariae were obtained from Ridgeway Research Ltd., (St Briavels, UK) or Baldwin Aquatics, Inc. (Monmouth, Oregon) and stored at $4{ }^{\circ} \mathrm{C}$ until use. Excystation of metacercariae was performed as described by Carmona et al. (1993) with minor modifications. NEJ were collected by pipetting and maintained in $38^{\circ} \mathrm{C}$ Roswell Park Memorial Institute medium (RPMI) 1640 medium (Gibco, Ireland) until infection.

\section{Tissue donors}

All procedures involving animal tissue were carried out following approval by the University College Dublin (UCD) Animal Research Ethics Committee (AREC-14-28). Twenty-three 6 weeks old male Wistar rats (250-300 g) were housed in the Biomedical Facility in UCD in controlled environmental conditions. The animals were euthanized by stunning, then cervical dislocation. Laparotomy was performed, the caecum exposed and the small intestine was identified. The portion of small intestine between 25 and $5 \mathrm{~cm}$ just before the cecum was dissected and kept in temperate incubation medium.

\section{Chamber configuration}

The method was based on modifications of a horizontal diffusion chamber developed for ex vivo bacterial studies (Soni et al. 2006). The chamber was composed of two sections: the basolateral and apical compartments. The basolateral compartment consisted of a half-filled $9 \mathrm{~mL}$ glass vacutainer tube. The apical compartment was either a $1.5 \mathrm{~mL}$ or $200 \mu \mathrm{L}$ eppendorf tube whose lid was drilled to create an aperture. NEJ were introduced into the chamber from the

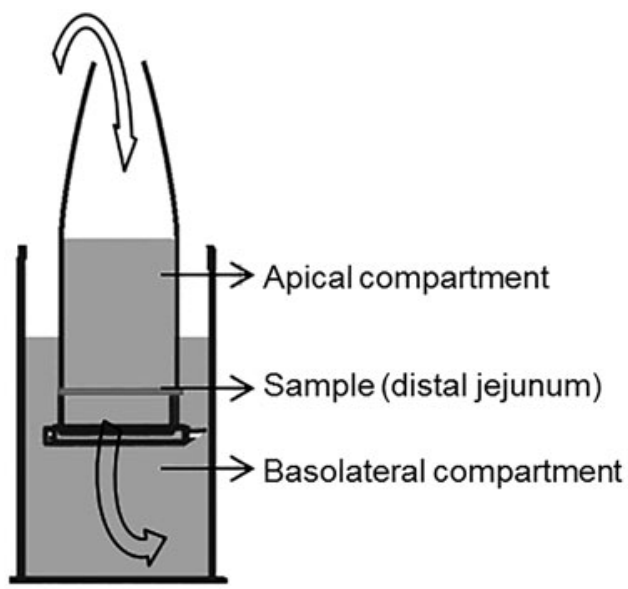

Fig. 1. Scheme of the modified horizontal diffusion chamber and the NEJ flow The Horizontal diffusion chamber consisted of the assembly of an apical and a basolateral compartment. The apical compartment was made from an eppendorf tube, the size of which was selected depending on the conditions employed for each method. The basolateral compartment consisted of medium half-filled $9 \mathrm{~mL}$ vacutainer tubes mounted inside a temperature controlled system. An aperture was drilled into the tip of the apical chamber to facilitate introduction of NEJ. The lid of the chamber was also drilled to create an aperture in which the tissue sample was mounted. NEJ that completely traversed through the jejunum were collected at the bottom of the basolateral compartment. Abbreviation: NEJ, newly excysted juvenile.

drilled tip side whereas the lid region was the area where NEJ came in contact with gut tissue. The apical compartment fitted into the basolateral compartment, with the tip region facing to the upper side of the basolateral compartment (Fig. 1).

\section{Tissue preparation, chamber assembly and NEF challenge}

Intestinal contents were removed by flushing with medium. The jejunum $(3-4 \mathrm{~cm})$ was opened along its mesenteric border to create a sheet which was pinned on a moistened dissection board with the serosal surface in contact with the board. The tissue was regularly irrigated with medium. Several adjacent sheets were obtained from each donor. The apical compartments of each chamber were opened and their lids were positioned between the corkboard and the serosal layer. The apical compartments were then closed making sure that the tissue covered the entire aperture. The chambers were then filled with medium. Tissue debris was removed and each chamber assembled. NEJ were added to the apical compartment using a $200 \mu \mathrm{L}$ pipette.

\section{Experimental conditions and assessment of migration in chambers}

Seven different protocols were evaluated, as described in Table 1. The conditions that varied 
Table 1. Parameters for the protocols assessed during method optimization

\begin{tabular}{|c|c|c|c|c|c|c|c|c|c|}
\hline Protocol & Donor specie & Sex & Age & Number animals & Chambers per time point & Chamber size (mL) & Medium & Temp $\left({ }^{\circ} \mathrm{C}\right)$ & Heating system \\
\hline 1 & Wistar Rat & Male & 6 w.o. & 3 & 3 & $1 \cdot 5$ & $5 \% \mathrm{CO}_{2}$ Krebs-Henseleit & 37 & Water bath \\
\hline 2 & Wistar Rat & Male & 6 w.o. & 4 & 4 & $1 \cdot 5$ & $5 \% \mathrm{CO}_{2}$ Krebs-Henseleit & 37 & Water bath \\
\hline 3 & Wistar Rat & Male & 6 w.o. & 2 & 2 & $0 \cdot 2$ & $5 \% \mathrm{CO}_{2}$ RPMI 1640 & 37 & Heating block \\
\hline 4 & Wistar Rat & Male & 6 w.o. & 4 & 4 & $0 \cdot 2$ & $5 \% \mathrm{CO}_{2}$ RPMI 1640 & 37 & Heating block \\
\hline 5 & Wistar Rat & Male & 6 w.o. & 4 & 4 & $0 \cdot 2$ & RPMI 1640 & 37 & Heating block \\
\hline 6 & Wistar Rat & Male & 6 w.o. & 4 & 6 & $0 \cdot 2$ & RPMI 1640 & 39 & Heating block \\
\hline 7 & Wistar Rat & Male & 6 w.o. & 3 & 18 & $0 \cdot 2$ & RPMI 1640 & 39 & Heating block \\
\hline Protocol & \multicolumn{2}{|c|}{ Metacercariae supplier } & \multicolumn{2}{|c|}{ Metacercariae storage time } & NEJ inoculation dose & $\begin{array}{l}\text { Accuracy of NEJ } \\
\text { inoculation dose }\end{array}$ & 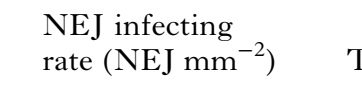 & Time points (min) & Histology \\
\hline 1 & \multicolumn{2}{|c|}{ Ridgeway research } & \multicolumn{2}{|c|}{ Less than 6 months } & $37 \cdot 38 \pm 5 \cdot 22$ & NO & $0 \cdot 48$ & $60,120,180,240$ & $\mathrm{NO}^{\mathrm{a}}$ \\
\hline 2 & \multicolumn{2}{|c|}{ Norm Baldwin } & \multicolumn{2}{|c|}{ Less than 6 months } & 40 & NO & $0 \cdot 51$ & $60,120,180,240$ & $\mathrm{NO}^{\mathrm{a}}$ \\
\hline 3 & \multicolumn{2}{|c|}{ Norm Baldwin } & \multicolumn{2}{|c|}{ More than 6 months } & $36 \pm 2 \cdot 77$ & $\mathrm{NO}$ & $1 \cdot 83^{2}$ & $30,60,90,120,150$ & YES \\
\hline 4 & \multicolumn{2}{|c|}{ Norm Baldwin } & \multicolumn{2}{|c|}{ Less than 6 months } & $31 \pm 8 \cdot 82$ & $\mathrm{NO}$ & $1 \cdot 58$ & $30,60,90,120,150$ & YES \\
\hline 5 & \multicolumn{2}{|c|}{ Norm Baldwin } & \multicolumn{2}{|c|}{ Less than 6 months } & $33 \pm 4 \cdot 95$ & $\mathrm{NO}$ & $1 \cdot 68$ & $30,60,90,120,150$ & YES \\
\hline 6 & \multicolumn{2}{|c|}{ Norm Baldwin } & \multicolumn{2}{|c|}{ Less than 6 months } & $42 \pm 3 \cdot 75$ & $\mathrm{NO}$ & $2 \cdot 14$ & $30,60,90,120,150$ & YES \\
\hline 7 & \multicolumn{2}{|c|}{ Norm Baldwin } & \multicolumn{2}{|c|}{ Less than 6 months } & $43 \cdot 56 \pm 1 \cdot 6$ & YES & $2 \cdot 21$ & $30,60,90,120,150$ & $\mathrm{NO}$ \\
\hline
\end{tabular}

NEJ, newly excysted juvenile; Temp, temperature; w.o., weeks old.

a NEJs counted under stereoscope with no distinction of their localization. 
were as follows: incubation medium, the supply of $\mathrm{CO}_{2}$ gas, size of apical compartment, incubation time, temperature, heating system and number of NEJ per chamber. Fluid from the apical compartment was collected carefully at each time point and placed in a watch glass. NEJ found here were counted using a stereoscope. In parallel, fluid from the basolateral compartment was allowed to settle for $10 \mathrm{~min}$ so that all NEJ sedimented to the bottom of the chamber before being counted.

\section{Assessment of NEF migration in distal jejunum: tissue embedding and histology staining}

In order to assess the NEJ pattern of migration in distal jejunum tissue samples were fixed separately in $10 \%$ buffered formalin (Fisher Chemical, UK) at each time point, using one of two approaches:

Methodology used for Protocols 1 and 2. After tissue fixation, specimens were placed on a microscope slide. Slides were viewed with an Olympus BX40FA camera (Olympus Optical Co, Ltd. Japan) using 4x and 20x Ach objectives (Olympus, Japan).

Methodology used for Protocols 3, 4, 5 and 6. Histological examination was performed following collection of semi-circular tissue samples. The tissue pieces were mounted into cassettes, processed and embedded in paraffin wax. Sections of $5 \mu \mathrm{m}$ thick were cut from the paraffin wax block. One in every ten cuts was mounted in slides and incubated at $58{ }^{\circ} \mathrm{C}$ overnight. Slides were dewaxed and haematoxylin and eosin $(\mathrm{H} \& \mathrm{E})$ staining was performed on all samples in a Shandon Gemini Varistain automatic slide stainer (Thermo Scientific). Slides were viewed as previously described. Localization (mucosa or serosa) and number of NEJ were recorded. NEJ located at the lumenal surface were considered as NEJ located in the mucosa.

\section{Accuracy of NEF infective dose}

NEJ were placed into the apical compartment by pipetting, and tips that were used for the NEJ challenge were kept and checked under a stereoscope. Numbers of NEJ that remained adhered in the tips were subtracted from the original infective dose to obtain an accurate NEJ inoculation dose.

\section{Methodology refinement and validation}

Once the conditions for NEJ migration had been optimized, the protocol was repeated to establish the kinetics of migration. Eighteen apical chambers were moved to fresh basolateral compartments every $30 \mathrm{~min}$ until $150 \mathrm{~min}$ of incubation. After $150 \mathrm{~min}$, fluid from the apical chamber was collected and NEJ found was counted as previously described. The fluid from each basolateral compartment was allowed to settle for $10 \mathrm{~min}$ and $\mathrm{NEJ}$ was counted as described.

\section{Graphs and statistical analysis}

Results were plotted using GraphPad Prism 5 software. Numbers of $\mathrm{NEJ}$ at each location and time point were expressed as means (士ts.D.). Data were analysed using the Friedman test and Dunn's multiple comparison test was employed to compare differences in migration between time points.

\section{RESULTS}

\section{Protocols 1, 2, 3 and 4}

The results of all the protocols employed are given in Table 2. Using Protocol 1, although NEJ were detected in the apical compartment at all the time points none were found in the basolateral compartment at any time point. On microscopic examination $15 \%$ of $\mathrm{NEJ}$ were detected in the tissue $180 \mathrm{~min}$ post-infection. However, the layers where the NEJ were located could not be distinguished. Changing the strain of metacercariae (Protocol 2) did not produce any increase in NEJ migration, suggesting that the incubation medium, the size of the chamber or the heating system were critical factors to consider. Changing these three factors (Protocol 3) did not result in any increase in NEJ counts in the basolateral compartment. However, there was a slight increase in the NEJ counts found within the gut tissue, with percentages that varied from $4 \cdot 13$ $( \pm 2 \cdot 02)$ to $26 \cdot 49 \%( \pm 18 \cdot 36)$. No significant differences were detected in NEJ counts from mucosal or serosal layers. Considering that this was the first time we were able to identify NEJ in the distal jejunum we concluded that using small chambers immersed in RPMI 1640 medium worked better than large chambers immersed in Krebs-Henseleit solution

The low infection rate led us to conclude that the viability of the metacercariae was too low as they had been stored for more than 6 months. In order to assure that metacercarial viability did not interfere with the assay, metacercariae less than 6 months old were used for the further optimization experiments. Using fresh metacercariae in the same conditions as Protocol 3 (Protocol 4) did increase NEJ counts in the basolateral region, with $5 \cdot 88 \%$ $( \pm 5 \cdot 61)$ of NEJ detected after $120 \mathrm{~min}$ incubation. However, the NEJ counts in histological sections were lower than in the previous method suggesting that further changes were necessary. We concluded that although standardizing metacercarial viability was an important variable to take into account, modification of other factors such as the incubation medium or the incubation temperature would be useful to investigate. 
Table 2. Comparison of newly excysted juvenile (NEJ) migration in vitro under the different protocols used in optimization experiments

\begin{tabular}{|c|c|c|c|c|c|c|c|c|c|c|c|c|c|c|c|c|}
\hline \multirow[b]{2}{*}{ Compartment } & \multirow[b]{2}{*}{$\begin{array}{l}\text { Time } \\
(\mathrm{min})\end{array}$} & \multicolumn{2}{|l|}{ Protocol 1} & \multicolumn{2}{|l|}{ Protocol 2} & \multirow[b]{2}{*}{$\begin{array}{l}\text { Time } \\
(\mathrm{min})\end{array}$} & \multicolumn{2}{|l|}{ Protocol 3} & \multicolumn{2}{|l|}{ Protocol 4} & \multicolumn{2}{|l|}{ Protocol 5} & \multicolumn{2}{|l|}{ Protocol 6} & \multicolumn{2}{|l|}{ Protocol 7} \\
\hline & & Mean \pm s.D. & $N$ & Mean \pm s.D. & $N$ & & Mean \pm s.D. & $N$ & Mean \pm s.D. & $N$ & Mean \pm s.D. & $N$ & Mean \pm s.D. & $N$ & Mean \pm s.D. & $N$ \\
\hline \multirow[t]{5}{*}{ Apical } & & & & & & 30 & $56 \cdot 99 \pm 57 \cdot 01$ & 2 & $73 \cdot 80 \pm 0.38$ & 2 & $47 \cdot 77 \pm 24 \cdot 19$ & 3 & $36 \cdot 95 \pm 16 \cdot 8$ & 6 & & \\
\hline & 60 & $31 \cdot 67 \pm 15 \cdot 28$ & 3 & $27 \cdot 33 \pm 14 \cdot 34$ & 3 & 60 & $21 \cdot 05$ & 1 & $42 \cdot 75 \pm 12 \cdot 07$ & 2 & $25 \cdot 11 \pm 14 \cdot 83$ & 3 & $36 \cdot 42 \pm 21 \cdot 08$ & 6 & & \\
\hline & 120 & $26 \cdot 67 \pm 6$ & 3 & $19 \cdot 08 \pm 22$ & 4 & 90 & $34 \cdot 22 \pm 11 \cdot 17$ & 2 & $56 \cdot 20 \pm 36 \cdot 26$ & 3 & $48 \cdot 25 \pm 15 \cdot 48$ & 3 & $38 \cdot 01 \pm 16 \cdot 41$ & 6 & & \\
\hline & 180 & $13 \cdot 33 \pm 15 \cdot 28$ & 3 & $9 \cdot 00 \pm 10 \cdot 52$ & 4 & 120 & $29 \cdot 20 \pm 14 \cdot 53$ & 2 & $52 \cdot 42 \pm 39 \cdot 07$ & 4 & $27 \cdot 17 \pm 15 \cdot 68$ & 4 & $40 \cdot 34 \pm 16 \cdot 01$ & 6 & & \\
\hline & 240 & $10 \pm 16 \cdot 02$ & 3 & $40 \pm 28 \cdot 28$ & 2 & 150 & $11 \cdot 00 \pm 4 \cdot 09$ & 2 & $57 \cdot 75 \pm 17 \cdot 69$ & 4 & $35 \cdot 41 \pm 15 \cdot 96$ & 3 & $20 \cdot 84 \pm 7 \cdot 99$ & 6 & $15 \cdot 93 \pm 6 \cdot 37$ & 3 \\
\hline \multirow[t]{5}{*}{ Basolateral } & & & & & & 30 & 0 & 2 & 0 & 2 & $1 \cdot 73 \pm 3$ & 3 & $2 \cdot 91 \pm 3 \cdot 68$ & 6 & $4 \cdot 14 \pm 3 \cdot 59$ & 3 \\
\hline & 60 & 0 & 3 & 0 & 3 & 60 & 0 & 1 & $1 \cdot 32 \pm 1 \cdot 86$ & 2 & $0 \cdot 83 \pm 1 \cdot 44$ & 3 & $13 \cdot 75 \pm 24 \cdot 38$ & 6 & $17 \cdot 56 \pm 5 \cdot 86$ & 3 \\
\hline & 120 & 0 & 3 & 0 & 4 & 90 & 0 & 2 & $1 \cdot 01 \pm 1 \cdot 75$ & 3 & $7 \cdot 62 \pm 7 \cdot 19$ & 3 & $14 \cdot 36 \pm 8 \cdot 21$ & 6 & $29 \cdot 26 \pm 7 \cdot 94$ & 3 \\
\hline & 180 & 0 & 3 & 0 & 4 & 120 & 0 & 2 & $5 \cdot 88 \pm 5 \cdot 61$ & 4 & $22 \cdot 73 \pm 3 \cdot 49$ & 4 & $7 \cdot 79 \pm 4 \cdot 74$ & 6 & $35 \cdot 55 \pm 5 \cdot 85$ & 3 \\
\hline & 240 & 0 & 3 & 0 & 2 & 150 & 0 & 2 & 0 & 4 & $16 \cdot 37 \pm 1 \cdot 97$ & 3 & $35 \cdot 23 \pm 15 \cdot 84$ & 6 & $40 \cdot 53 \pm 7 \cdot 17$ & 3 \\
\hline \multirow[t]{5}{*}{ Mucosa } & & & & & & 30 & $4 \cdot 13 \pm 2 \cdot 02$ & 2 & 0 & 2 & $7 \cdot 69 \pm 10 \cdot 88$ & 3 & $10 \cdot 32 \pm 5 \cdot 75$ & 6 & & \\
\hline & 60 & & & & & 60 & $5 \cdot 26$ & 1 & $1 \cdot 32 \pm 1 \cdot 86$ & 2 & $9 \cdot 88 \pm 8 \cdot 87$ & 3 & $14.92 \pm 7 \cdot 59$ & 6 & & \\
\hline & 120 & & & & & 90 & $9 \cdot 21 \pm 1 \cdot 87$ & 2 & 0 & 3 & $3 \cdot 81 \pm 3 \cdot 3$ & 3 & $12 \cdot 35 \pm 7 \cdot 17$ & 6 & & \\
\hline & 180 & & & & & 120 & $15 \cdot 87 \pm 14 \cdot 79$ & 2 & $0 \cdot 68 \pm 1 \cdot 35$ & 4 & $4 \cdot 29 \pm 4.96$ & 4 & $21 \cdot 94 \pm 17 \cdot 3$ & 6 & & \\
\hline & 240 & & & & & 150 & $2 \cdot 71 \pm 3 \cdot 83$ & 2 & $6 \cdot 76 \pm 7 \cdot 8$ & 4 & $4 \cdot 14 \pm 4 \cdot 6$ & 3 & $7 \cdot 05 \pm 6$ & 6 & & \\
\hline \multirow[t]{5}{*}{ Serosa } & & & & & & 30 & 0 & 2 & 0 & 2 & $5 \cdot 77 \pm 8 \cdot 16$ & 3 & $0 \cdot 82 \pm 1 \cdot 28$ & 6 & & \\
\hline & 60 & & & & & 60 & $2 \cdot 63$ & 1 & 0 & 2 & $3 \cdot 13 \pm 3 \cdot 49$ & 3 & $5.42 \pm 6.79$ & 6 & & \\
\hline & 120 & & & & & 90 & $5 \cdot 26 \pm 3 \cdot 72$ & 2 & $4 \cdot 04 \pm 7$ & 3 & $6 \cdot 67 \pm 7 \cdot 19$ & 3 & $2 \cdot 50 \pm 3 \cdot 87$ & 6 & & \\
\hline & 180 & & & & & 120 & $10 \cdot 64 \pm 3 \cdot 57$ & 2 & 0 & 4 & $4 \cdot 87 \pm 4 \cdot 84$ & 4 & $2 \cdot 88 \pm 4 \cdot 51$ & 6 & & \\
\hline & 240 & & & & & 150 & $10 \cdot 89 \pm 7 \cdot 54$ & 2 & $3 \cdot 93 \pm 4 \cdot 61$ & 4 & $16 \cdot 37 \pm 1 \cdot 97$ & 3 & $2 \cdot 06 \pm 3 \cdot 3$ & 6 & & \\
\hline Total distal & & & & & & 30 & $4 \cdot 13 \pm 2 \cdot 02$ & 2 & 0 & 2 & $13 \cdot 46 \pm 19 \cdot 04$ & 3 & $11 \cdot 14 \pm 5 \cdot 61$ & 6 & & \\
\hline Jejunum & 60 & $6 \cdot 67 \pm 2 \cdot 89$ & 3 & $4 \cdot 44 \pm 7 \cdot 7$ & 3 & 60 & $7 \cdot 89$ & 1 & $1 \cdot 32 \pm 1 \cdot 86$ & 2 & $13 \cdot 01 \pm 5 \cdot 4$ & 3 & $20 \cdot 33 \pm 11 \cdot 5$ & 6 & & \\
\hline (MUCO + & 120 & $11 \cdot 67 \pm 8$ & 3 & $19 \cdot 17 \pm 22$ & 4 & 90 & $14 \cdot 48 \pm 5 \cdot 58$ & 2 & $4 \cdot 04 \pm 7$ & 3 & $10 \cdot 48 \pm 4 \cdot 37$ & 3 & $14 \cdot 85 \pm 8 \cdot 2$ & 6 & & \\
\hline \multirow[t]{2}{*}{ SERO) } & 180 & $15 \pm 10$ & 3 & $5 \pm 10$ & 4 & 120 & $26 \cdot 49 \pm 18 \cdot 36$ & 2 & $0 \cdot 68 \pm 1 \cdot 35$ & 4 & $9 \cdot 16 \pm 9 \cdot 37$ & 4 & $24 \cdot 82 \pm 19 \cdot 01$ & 6 & & \\
\hline & 240 & $0 \pm 3 \cdot 85$ & 3 & 0 & 2 & 150 & $13 \cdot 59 \pm 11 \cdot 36$ & 2 & $10 \cdot 69 \pm 12 \cdot 11$ & 4 & $20 \cdot 51 \pm 6 \cdot 51$ & 3 & $9 \cdot 12 \pm 8 \cdot 44$ & 6 & & \\
\hline NEJ detection & & & & & & & $45 \cdot 46 \pm 28 \cdot 07$ & 9 & $62 \cdot 12 \pm 23 \cdot 37$ & 15 & $59 \cdot 03 \pm 18 \cdot 74$ & 16 & $65 \cdot 37 \pm 20 \cdot 42$ & 30 & $61 \cdot 51 \pm 18 \cdot 12$ & 18 \\
\hline
\end{tabular}

Results expressed in percentage (\%). 


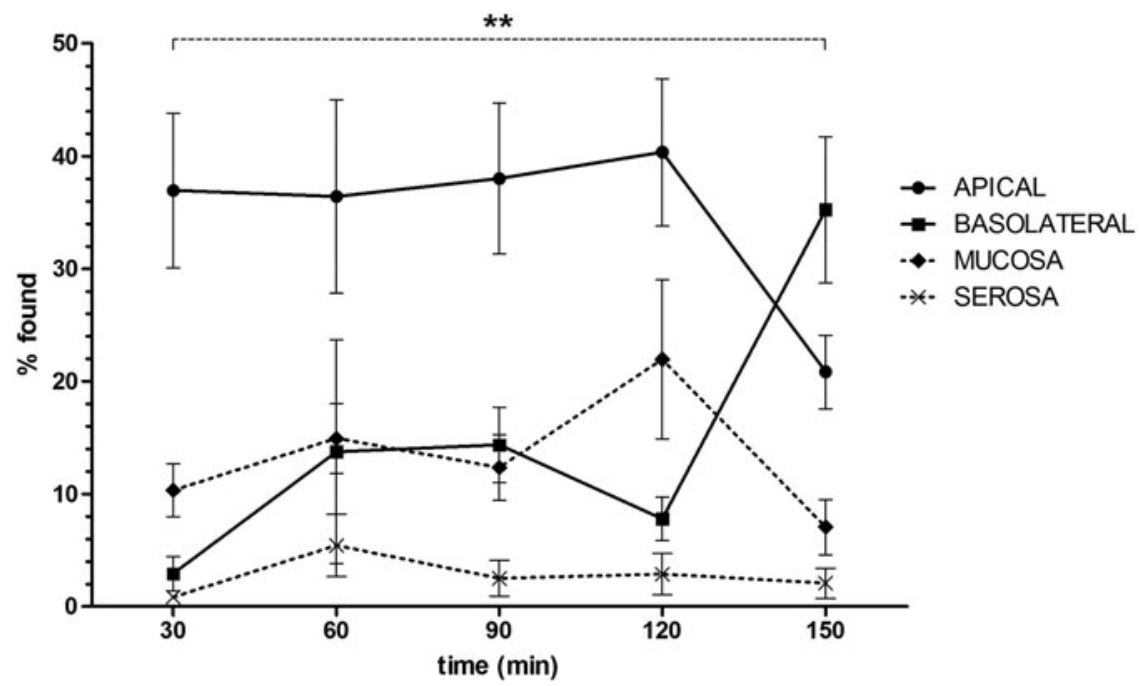

Fig. 2. Kinetics of NEJ invasion in rat distal jejunum using Protocol 6 NEJ were inoculated into the in vitro system as described and allowed to migrate through rat distal jejunum for 30-150 min. NEJ located in apical (continuous line circles) and basolateral (continuous line squares) were counted. Jejunum was fixed and serial histological examination was performed every $50 \mu \mathrm{m}$ to assess the percentage of NEJ located in the mucosa (dashed line diamonds) and serosa (dashed line crosses) at the different time points. Total percentages were calculated according to the initial NEJ inoculation dose. Results were calculated based on six independent experiments. Vertical bars represent S.E. Asterisks represent significant differences in the percentage of $\mathrm{NEJ}$ found in the basolateral chamber between time points $(* * P \leqslant 0 \cdot 01)$. Abbreviation: NEJ, newly excysted juvenile.

The influence of incubation medium and incubation temperature were the key factors that triggered NEF invasion into the basolateral compartment

The following protocols were modified from the previous ones in two respects. For the first modification, which corresponded to Protocol 5, RPMI 1640 medium was no longer gassed with $5 \% \mathrm{CO}_{2}$. This protocol showed for the first time a continuous migration of the NEJ from the apical to the basolateral chamber at all the time points, with values of $22.73 \%$ $( \pm 3 \cdot 49)$ and $16 \cdot 37 \%( \pm 1.97)$ at 120 and $150 \mathrm{~min}$, respectively. In addition, the NEJ counts in the distal jejunum were higher than the NEJ counts found in the previous protocol. We concluded that the lack of additional $\mathrm{CO}_{2}$ supplied to the medium was responsible for these differences.

In spite of this increase in migration, $\mathrm{NEJ}$ migration at $150 \mathrm{~min}$ was lower than the NEJ migration detected by other in vitro models (Hanna and Ballawy, 1975; Van Milligen et al. 1998a, b; McGonigle et al. 2008). For that reason, we performed Protocol 6. This was based on Protocol 5 with the modification of increasing the temperature of the system from 37 to $39^{\circ} \mathrm{C}$. As shown in Fig. 2, the percentage of NEJ found in the apical compartment was stable from time 30 to $120 \mathrm{~min}$, this value varying between $36.42 \%$ $( \pm 21 \cdot 08)$ and $40 \cdot 34 \%( \pm 16 \cdot 01)$. After $120 \mathrm{~min}$, a large decrease of NEJ was observed in the apical compartment, of the order of $20 \cdot 84 \% \quad( \pm 7 \cdot 99)$. The number of NEJ observed in the basolateral compartment increased over time, with $150 \mathrm{~min}$ the time point with most NEJ found in this chamber $(35 \cdot 23$ $\pm 15 \cdot 84 \%)$. This was the first time where we observed a significant difference in NEJ migration between 30 and 150 min with a final NEJ migration rate comparable with previous studies. We concluded that an increase of $2{ }^{\circ} \mathrm{C}$ in the incubation temperature provided this improvement.

There were no significant differences in the NEF counts in mucosa and serosa in Protocol 6

The percentage of NEJ found in the mucosa (Fig. 2) increased at all the time points, and peaked at 120 min post infection at $21 \cdot 94 \%( \pm 17 \cdot 29)$. After this peak, a drop in this percentage was observed at 150 min post infection which correlates with the increase of the NEJ detected previously in the basolateral compartment. There were no significant differences between the counts at 30 and $150 \mathrm{~min}$. Fewer than $6 \% \mathrm{NEJ}$ were detected in the serosa, with $5 \cdot 41 \%( \pm 6 \cdot 78)$ the highest value at $60 \mathrm{~min}$ post infection. Examples of NEJ located in the mucosa and serosa are shown in Fig. 3.

\section{NEF detection methods of all the previous protocols underestimated the true NEF counts}

Taking into account the NEJ counts in the apical and basolateral compartments and within the gut in all the protocols assessed, the highest level of NEJ detection, as a percentage of NEJ inoculated, was seen in Protocol 6 with an average of $65 \cdot 37 \%$ $( \pm 20 \cdot 42)$ suggesting that significant numbers of 

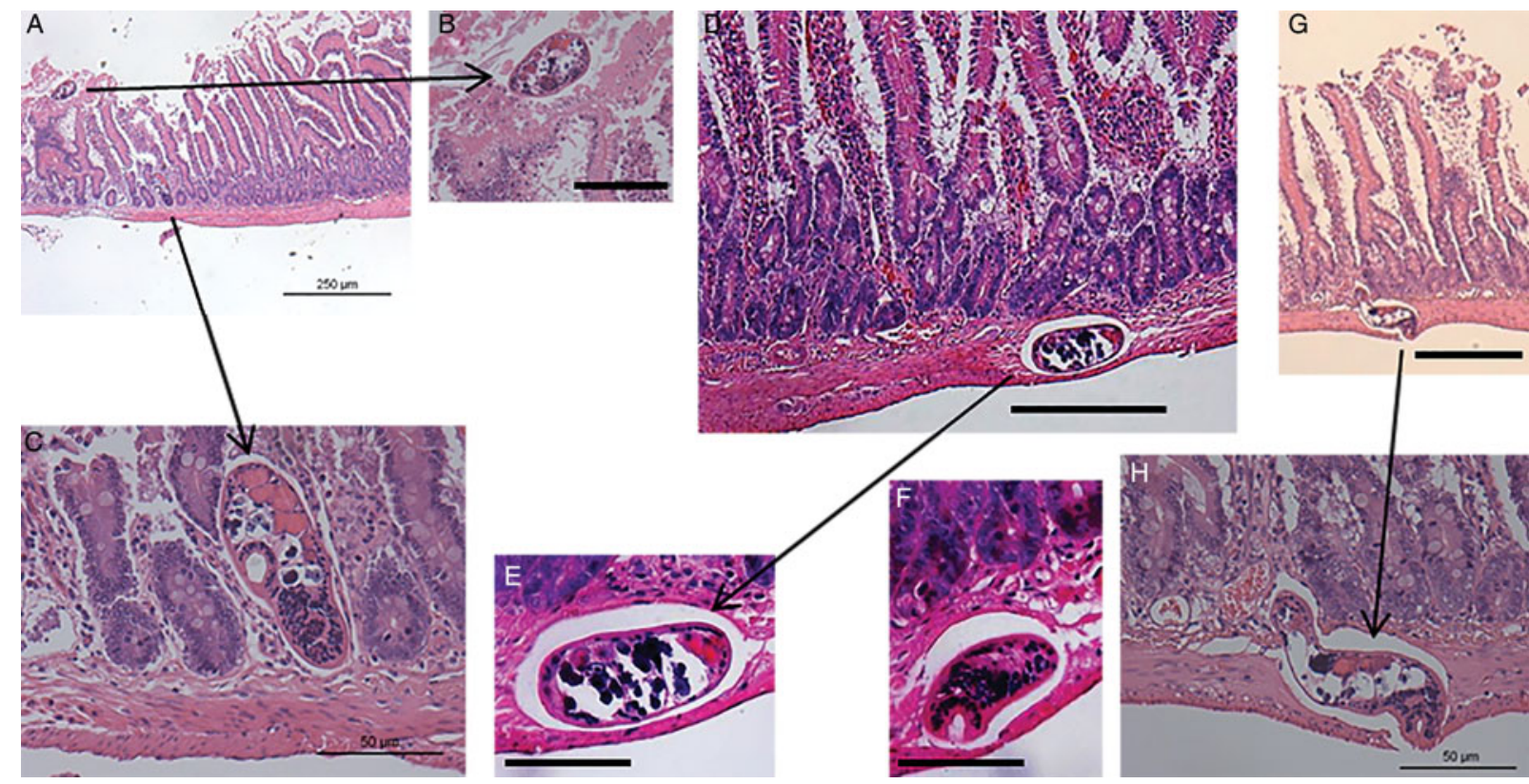

Fig. 3. Detection and localization of NEJ in rat distal jejunum by histology NEJ were inoculated into the in vitro system as described and allowed to migrate through rat distal jejunum. Jejunum was fixed and the presence of NEJ in the mucosa $(\mathrm{A}, \mathrm{B}, \mathrm{C})$ and in the serosa $(\mathrm{D}, \mathrm{E}, \mathrm{F}, \mathrm{G}, \mathrm{H})$ was assessed every $50 \mu \mathrm{m}$ of tissue after $\mathrm{H} \& \mathrm{E}$ staining at $60 \mathrm{~min}(\mathrm{~A}, \mathrm{~B}, \mathrm{C})$, $90 \mathrm{~min}(\mathrm{D}, \mathrm{E}), 120 \mathrm{~min}(\mathrm{~F})$ and at $150 \mathrm{~min}(\mathrm{G}, \mathrm{H})$ post NEJ inoculation. Scale bar $=250 \mu \mathrm{m}(\mathrm{A}, \mathrm{G})$. Scale bar $=50 \mu \mathrm{m}$ (B, C, D, E, F, H). Abbreviations: H\&E, haematoxylin and eosin; NEJ, newly excysted juvenile.

NEJ were missing and/or that the NEJ infecting dose could have been overestimated. In order to investigate this and to obtain a more accurate NEJ migration curve, Protocol 7 was designed.

\section{Protocol 7 reproduced the kinetics seen in Protocol 6 and also increased the NEF detection rate in the basolateral compartment by $40.53 \%$}

Protocol 7 was carried out taking into account the possibility of overestimating the initial NEJ dose inoculated into the chambers. We found that $7 \cdot 3 \%$ $( \pm 3 \cdot 01)$ of the NEJ remained attached to the tips that were used for infecting the chambers, thus confirming the initial overestimation of the NEJ dose in previous protocols.

Once we calculated the exact NEJ dose we wanted to reproduce and obtain a more realistic cumulative NEJ invasion curve using a larger number of chambers. Our results confirmed the reproducibility of the method counting NEJ in the basolateral compartment at all the time points (Fig. 4). Although the highest percentage of NEJ in the basolateral compartment was detected at $150 \mathrm{~min}(40.53 \pm$ $7 \cdot 17)$ significant differences were already observed in NEJ numbers from 30 to 90 min post infection. In addition, NEJ counts in the apical chambers decreased from $20 \cdot 84 \%$ in Protocol 6 to $15.93 \%$ $( \pm 6 \cdot 37)$ in the current protocol suggesting that more NEJ were invading the mucosa and serosa. Although histology was not performed and NEJ counts in the jejunum were not used for assessing this protocol the percentage of NEJ detection from the tips, the apical and the basolateral compartment only (i.e. excluding NEJ within the tissue) was $61 \cdot 51 \%$.

\section{DISCUSSION}

We aimed to develop an optimized robust and reproducible in vitro model for assessing $F$. hepatica NEJ migration. Sheets of isolated intestine have been used successfully to examine pathogen entry (Baird et al. 2004) as well as ion transport responses of previously sensitized mucosal sheets to specific antigen (Baird and O'Malley, 1993). We modified the structure of the horizontal diffusion chamber used in previous microbiological studies (Soni et al. 2006) to our requirements for parasite studies. Optimization of the method was carried out by investigating seven different protocols to assess in vitro NEJ invasion under physiological conditions. Protocols 5, 6 and 7 showed active NEJ migration. We observed that incubation temperature, incubation medium and chamber size/rate of infection were the most important parameters in determining migration from apical to basolateral compartment. Temperature also correlated with the efficiency of the heating system. When a water bath was used as the heating system, variability due to vaporization, room temperature changes etc. could mitigate against the correct temperature being reached. However, the use of a heating block to surround the whole basolateral chamber eliminated these factors. Because steel has a higher thermal conductivity than water $\left(52 \mathrm{~W} \mathrm{mK}^{-1}\right.$ for steel vs $0.6 \mathrm{~W} \mathrm{mK}^{-1}$ for water at 


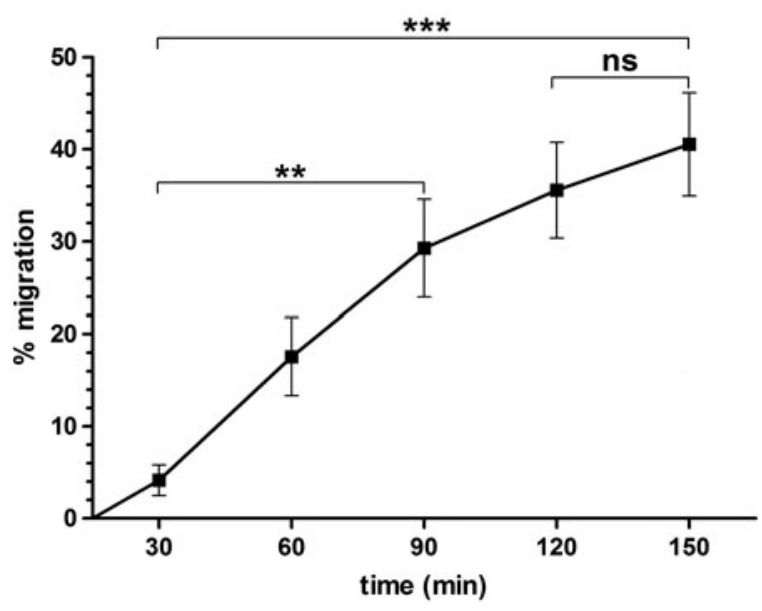

Fig. 4. Kinetics of NEJ invasion in rat distal jejunum using Protocol 7 NEJ were inoculated into the in vitro system as described and allowed to migrate through rat distal jejunum from $30-150 \mathrm{~min}$. A total of 18 chambers were used in the confirmation of the method. NEJ located in basolateral compartments were counted and the total percentage was calculated after recalculation of the NEJ inoculation dose. Results were calculated based on three independent experiments. Vertical bars represent s.E. Asterisks represents significant differences in the percentage of NEJ found in the basolateral compartment between different time points (** $P \leqslant 0 \cdot 01, * * * P \leqslant 0 \cdot 001)$. Abbreviation: NEJ, newly excysted juvenile.

$20^{\circ} \mathrm{C}$, (Young, 1992), heat is conducted more efficiently, reaching the desired temperature very quickly. Because of the higher percentage of NEJ detected in the basolateral compartment in Protocols 6 and 7, we concluded that $39^{\circ} \mathrm{C}$ is the most reliable temperature to maximize NEJ migration in this system.

Chamber size and rate of infection are complementary factors. Because the number of NEJ used in each chamber was very similar (40 NEJ per chamber approximately), the size of the chamber determined the area of the sample, being larger in the $1.5 \mathrm{~mL}$ chambers and smaller in the $200 \mu \mathrm{L}$ chambers. Although other authors proposed that rate of infection does not interfere with the percentage of migration (Van Milligen et al. 1998a, b), these calculations were based on a gut loop model, by using a bigger portion of intestine. By using the gut loop model, homogeneity of NEJ distribution on the lumen surface is not guaranteed because it is possible that a considerable proportion of $\mathrm{NEJ}$ are crowded unevenly in some focal portions of the loop and the rest of the NEJ disperse in the rest of the sample. In our methods, because of the size of the chamber, a homogenous dispersion of NEJ is more likely, especially with the smaller compartment.

We observed that the incubation medium used had a considerable effect on NEJ migration. This is consistent with what has been described previously (Hanna and Ballawy, 1975). We found that RPMI 1640 without $\mathrm{CO}_{2}$ was the best medium to use in the current method. Carbon dioxide is typically used in mammalian tissue and cells for maintaining constantly a neutral-acid pH of the medium where cells are cultured for periods of days or weeks. It has been shown that the $\mathrm{pH}$ detected in the lumen of jejunum and ileum in rats tends to be quite high ( $\mathrm{pH}=7.9$ ) (Ward and Coates, 1987). Therefore, it can be suggested that, under these experimental conditions, $\mathrm{CO}_{2}$ gasification is not a crucial factor for NEJ and jejunum survival. In the present study, distal jejunum was cultured for less than $3 \mathrm{~h}$. As the $\mathrm{pH}$ indicator did not vary from the beginning to the end of the experimental period, we conclude that this was not long enough to change the $\mathrm{pH}$ to non-physiological conditions. The use of bile as a supplement to enhance migration was considered as it has been described that it is a direct stimulus for NEJ to leave the intestine (Tielens et al. 1981). Nevertheless, bile is also detrimental to NEJ survival and changes the NEJ glycocalyx (Bennett and Threadgold, 1975), which it is desirable to keep intact for assessing host-parasite interaction.

Taking into account the NEJ numbers found at apical and basolateral compartments in Protocols 6 and 7 we can confidently conclude that active migration was observed. Particularly in Protocol 7, there were significant differences in the NEJ migration between 30 and $90 \mathrm{~min}$ post-infection. This could explain the requirement for NEJ to recognize receptors in the gut as a trigger for the upregulation of the expression and secretion of active molecules such as cathepsin L3 and cathepsin Bs (Cancela et al. 2008; Robinson et al. 2009) and start migration very quickly in order to avoid host digestion or expulsion from the host. The $40 \cdot 53 \%$ migration rate observed after $150 \mathrm{~min}$ is comparable with migration observed in another ex vivo method where the same value was obtained in cumulative penetration at that time point (Van Milligen et al. 1998a, b), with another in vitro model created for $F$. gigantica invasion in distal ileum (Hanna and Ballawy, 1975) and with Japanese $F$. hepatica in rats (Kawano et al. 1991). Regarding the localization of the NEJ in the intestine at the different gut layers we found no significant differences between mucosa at serosa in Protocol 6 at any time point. For that reason histological examination was not performed for Protocol 7.

In the first attempt at optimization, $65 \cdot 37 \%$ of total NEJ were detected between apical and basolateral compartments along with in the gut tissue. The fact that this value was lower than that reported for an ex vivo model (Van Milligen et al. 1998a, b) made us consider that further improvements were required in NEJ identification to achieve an even higher rate of detection. After verifying with Protocol 7 that the dose of infection used in each chamber was actually a bit lower than originally calculated, because some NEJ were attached in the tip of the pipette, we obtained very similar NEJ detection rates. Although histological examinations were not 
performed in this last method we suggest that NEJ detection was improved compared with Protocol 6.

The method we used for the detection of NEJ within host tissue was based on previous work (Van Milligen et al. 1998a, b). Using our pattern of tissue screening, which is to examine every $50 \mu \mathrm{m}$, some NEJ located in tissue may have been hidden due to their orientation. As the images indicate, NEJ migrated actively in the tissue, with the oral sucker facing the gut tissue. When NEJs reached the serosal layer, they appeared to be surrounded by an empty cavity. As the serosa is a distinct barrier, we hypothesized that the cavity may be filled with ES products (Robinson et al. 2009; Cancela et al. 2010). However, since tissues were fixed by formalin, we cannot rule out the possibility that these were artefacts.

A limitation of this method is the progressive deterioration in the integrity of the mucosal tissue, as judged histologically, after $150 \mathrm{~min}$, thus limiting the time post-infection that can be investigated. Nevertheless, considering that mucosa was only compromised after $150 \mathrm{~min}$ and that no significant differences were detected in the migration of the NEJ between 120 and $150 \mathrm{~min}$ we concluded that this limitation did not interfere with our experiments.

All the modifications carried out during this optimization have led to the development of a reliable $e x$ vivo, method which is in accordance with the $3 \mathrm{Rs}$ (replacement, reduction and refinement) principle as no invasive procedures are needed and the number of animals required is minimized. The main advantage is that various experimental conditions can be applied to specimens from the same donor, which avoids the use of one animal for a single experimental condition, thus reducing intra animal variability. In addition, it is versatile as it can be applied to all the intestinal segments and to different animal species. Up to 12 chambers can be mounted from a single rat jejunum in the optimal time frame for assuring tissue integrity (data not shown). However, it is noteworthy to point out that the intestinal tract and the animal species chosen in the study are the key factors that determine the number of chambers that are possible to produce. In order to acquire statistically robust data, the number of chambers required for each experimental condition is dependent on the research question as well as other factors such as the power of the analysis and the confidence interval required, among others. Based on the data obtained in this study, we recommend that for similar work no less than three chambers per experimental condition and donor should be used.

In conclusion, we can say that the modified horizontal diffusion system we have developed and validated is a useful tool for assessment of gut penetration by $F$. hepatica NEJ in vitro. Multiple, matched tissues may be obtained from a single donor which permits good experimental design. This will be particularly useful in determining the migration pattern of NEJ that have been exposed for example to vaccine induced antibodies, glycan ligands, host lectin receptor agonists and putative chemotherapeutic agents. This method in combination with other in vitro methods will allow us to improve understanding of the main factors that contribute to the earliest stages of fasciolosis in the definitive host, and ultimately to increase the range of control methods available for this important parasitic disease.

\section{ACKNOWLEDGEMENTS}

We are grateful to Patsy Kearns and Fiona McCartney for animal care and preparation before euthanasia. We also thank Joseph Brady and Margot Coady for their assistance during the histology process.

\section{FINANCIAL SUPPORT}

This work was supported by the European Community's Seventh Framework Programme (PARAVAC - Project Number 265862 to G.M.).

\section{REFERENCES}

Baird, A. W. and O'Malley, K. E. (1993). Epithelial ion transport - possible contribution to parasite expulsion. Parasitology Today 9, 141-143. Baird, A. W., Campion, D. P., O'Brien, L. and Brayden, D. J. (2004). Oral delivery of pathogens from the intestine to the nervous system. Fournal of Drug Targeting 12, 71-78.

Bennett, C. E. and Threadgold, L. T. (1975). Fasciola hepatica: development of tegument during migration in mouse. Experimental Parasitology 38, 38-55.

Cancela, M., Acosta, D., Rinaldi, G., Silva, E., Durán, R., Roche, L., Zaha, A., Carmona, C. and Tort, J. F. (2008). A distinctive repertoire of cathepsins is expressed by juvenile invasive Fasciola hepatica. Biochimie $\mathbf{9 0}$, 1461-1475.

Cancela, M., Ruétalo, N., Dell'Oca, N., da Silva, E., Smircich, P., Rinaldi, G., Roche, L., Carmona, C., Alvarez-Valín, F., Zaha, A. and Tort, J. F. (2010). Survey of transcripts expressed by the invasive juvenile stage of the liver fluke Fasciola hepatica. BMC Genomics 11, 227. Carmona, C., Dowd, A. J., Smith, A. M. and Dalton, J. P. (1993). Cathepsin L proteinase secreted by Fasciola hepatica in vitro prevents antibody-mediated eosinophil attachment to newly excysted juveniles. Molecular and Biochemical Parasitology 62, 9-17.

Doy, T. G., Hughes, D. L. and Harness, E. (1981). Hypersensitivity in rats infected with Fasciola hepatica: possible role in protection against a challenge infection. Research in Veterinary Science 30, 360-363.

Hanna, R. E., Ballawy, S. S. and Jura, W. (1975). Methods for in vitro study of the invasive processes of Fasciola gigantica. Research in Veterinary Science 19, 96-97.

Kawano, J., Yamamoto, T., Koga, M., Shimizu, A. and Kimura, S. (1991). Penetration in vitro of newly excysted juvenile flukes of Japanese Fasciola sp. through ligated intestines of rabbits, mice, rats and chickens. Fournal of Veterinary Medical Science 54, 69-73.

McGonigle, L., Mousley, A., Marks, N. J., Brennan, G. P., Dalton, J. P., Spithill, T. W., Day, T. A. and Maule, A. G. (2008). The silencing of cysteine proteases in Fasciola hepatica newly excysted juveniles using RNA interference reduces gut penetration. International Fournal for Parasitology 38, 149-155.

Robinson, M. W., Menon, R., Donnelly, S. M., Dalton, J.P. and Ranganathan, S. (2009). An integrated transcriptomics and proteomics analysis of the secretome of the helminth pathogen Fasciola hepatica: proteins associated with invasion and infection of the mammalian host. Molecular and cellular Proteomics: MCP 8, 1891-1907.

Schumacher, W. (1938). Untersuchungen uber den Wanderungsweg und die Entwicklung von Fasciola hepatica L. im Endwirt. Zeitschrift fur Parasitenkunde 10, 608-643.

Soni, J., Baird, A. W., O'Brien, L. M., McElroy, M., Callanan, J. J., Bassett, H. F., Campion, D. and Brayden, D. J. (2006). Rat, ovine and 
bovine Peyer's patches mounted in horizontal diffusion chambers display sampling function. Fournal of Controlled Release: Official Fournal of the Controlled Release Society 115, 68-77.

Sukhdeo, M. V. and Mettrick, D. F. (1986). The behavior of juvenile Fasciola hepatica. Fournal of Parasitology 72, 492-497.

Tielens, A. G., Van der Meer, P. and Van den Bergh, S. G. (1981). Fasciola hepatica: simple, large-scale, in vitro excystment of metacercariae and subsequent isolation of juvenile liver flukes. Experimental Parasitology $\mathbf{5 1}, 8-12$.

Van Milligen, F. J., Cornelissen, J. B. and Bokhout, B. A. (1998a).

Location of induction and expression of protective immunity against Fasciola hepatica at the gut level: a study using an ex vivo infection model with ligated gut segments. Fournal of Parasitology 84, 771-777.
Van Milligen, F. J., Cornelissen, J. B., Gaasenbeek, C.P. and Bokhout, B. A. (1998b). A novel ex vivo rat infection model to study protective immunity against Fasciola hepatica at the gut level. Fournal of Immunological Methods 213, 183-190.

Van Milligen, F. J., Cornelissen, J. B. and Bokhout, B. A. (1999)

Protection against Fasciola hepatica in the intestine is highly correlated with eosinophil and immunoglobulin G1 responses against newly excysted juveniles. Parasite Immunology 21, 243-251.

Ward, F. W. and Coates, M. E. (1987). Gastrointestinal pH measurement in rats: influence of the microbial flora, diet and fasting. Laboratory Animals 21, 216-222.

Young, H. D. (1992). University Physics, 7th Edn. Addison Wesley, Reading, MA. 